\title{
Glutathione mediated regulation of oligomeric structure and functional activity of Plasmodium falciparum glutathione
} S-transferase

\author{
Timir Tripathi ${ }^{1}$, Stefan Rahlfs ${ }^{2}$, Katja Becker*2 and Vinod Bhakuni*1
}

Address: ${ }^{1}$ Division of Molecular and Structural Biology, Central Drug Research Institute, Lucknow 226001, India and ${ }^{2}$ Interdisciplinary Research Center, Justus-Liebig-University, Heinrich-Buff-Ring 26-32, 35392 Giessen, Germany

Email: Timir Tripathi - timir_biotech@rediffmail.com; Stefan Rahlfs - srahlfs@hotmail.com; Katja Becker* - becker.katja@gmx.de;

Vinod Bhakuni* - bhakuniv@rediffmail.com

* Corresponding authors

Published: 17 October 2007

BMC Structural Biology 2007, 7:67 doi:10.1 186/1472-6807-7-67
Received: 27 April 2007

Accepted: 17 October 2007

This article is available from: http://www.biomedcentral.com/1472-6807/7/67

(c) 2007 Tripathi et al; licensee BioMed Central Ltd.

This is an Open Access article distributed under the terms of the Creative Commons Attribution License (http://creativecommons.org/licenses/by/2.0), which permits unrestricted use, distribution, and reproduction in any medium, provided the original work is properly cited.

\begin{abstract}
Background: In contrast to many other organisms, the malarial parasite Plasmodium falciparum possesses only one typical glutathione $S$-transferase. This enzyme, PfGST, cannot be assigned to any of the known GST classes and represents a most interesting target for antimalarial drug development. The PfGST under native conditions forms non-covalently linked higher aggregates with major population ( $98 \%$ ) being tetramer. However, in the presence of $2 \mathrm{mM} \mathrm{GSH}$, a dimer of PFGST is observed. Recently reported study on binding and catalytic properties of PfGST indicated a GSH dependent low-high affinity transition with simultaneous binding of two GSH molecules to PfGST dimer suggesting that GSH binds to low affinity inactive enzyme dimer converting it to high affinity functionally active dimer. In order to understand the role of GSH in tetramer-dimer transition of PFGST as well as in modulation of functional activity of the enzyme, detailed structural, functional and stability studies on recombinant PFGST in the presence and absence of GSH were carried out.
\end{abstract}

Results: Our data indicate that the dimer - and not the tetramer - is the active form of PfGST, and that substrate saturation is directly paralleled by dissociation of the tetramer. Furthermore, this dissociation is a reversible process indicating that the tetramer-dimer equilibrium of PfGST is defined by the surrounding GSH concentration. Equilibrium denaturation studies show that the PFGST tetramer has significantly higher stability compared to the dimer. The enhanced stability of the tetramer is likely to be due to stronger ionic interactions existing in it.

Conclusion: This is the first report for any GST where an alteration in oligomeric structure and not just small conformational change is observed upon GSH binding to the enzyme. Furthermore we also demonstrate a reversible mechanism of regulation of functional activity of Plasmodium falciparum glutathione S-transferase via GSH induced dissociation of functionally inactive tetramer into active dimers. 


\section{Background}

Development of resistance to the drugs used for prophylaxis and treatment of malaria makes the identification and characterization of novel drug targets necessary $[1,2]$. Glutathione S-transferases (GSTs ${ }^{1}$; EC 2.5.1.18) form a family of phase II detoxification enzymes and are present in virtually all organisms. GSTs catalyze the nucleophilic addition of the tripeptide glutathione to a large variety of nonpolar compounds, thereby neutralizing their electrophilic sites and rendering the products more water soluble [3]. These products can be more easily excreted, usually as a part of the mercapturic acid pathway [4]. GSTs can furthermore detoxify lipid peroxidation products $[5,6]$ and serve as carrier proteins, so called ligandins, of certain organic molecules, which lead to the inactivation and immobilization of these compounds [4]. Other functions include the detoxification of hydroperoxides and the isomerization of specific metabolites (like prostaglandin $\mathrm{H}$ and 13- cis retinoic acid), reduction of dehydroascorbate and transfer of thiols [7] contributing to cellular signaling, regulation of transcription and stress response [8]. In cancer chemotherapy, the ability of GST to produce reactive metabolites has been exploited to target tumors that overexpress particular transferases [9].

On the basis of sequence similarity, immunological cross reactivity and substrate specificity, the cytosolic GSTs have been grouped into at least 13 classes: alpha, mu, pi, theta, sigma, kappa, omega, lambda, phi, tau, delta, zeta, and dehydroascorbate reductase (DHAR) [4,8,10-13]. Although the recognition of glutathione is conserved among different classes, the active site residues involved in glutathione binding vary from tyrosine in alpha, mu and pi classes (mammals) to serine in theta and zeta (ubiquitous), phi and tau (plants), and delta (insects) classes or cysteine in omega (mammals \& insects), beta (bacteria) and lambda classes as well as in DHAR (plants) [13].

GST of the malarial parasite Plasmodium falciparum represents a novel GST isoform, which cannot be assigned to any of the known GST classes and constitutes more than $1 \%$ of the total cellular protein of the parasite $[14,15]$. PfGST shares highest sequence similarities with pi-class GSTs from Dirofilaria immitis and Onchocerca volvolus ( $35 \%$ identity). GST activity has been reported in all Plasmodium species studied so far as well as in all intraerythrocytic stages of the parasite [16]. Furthermore GST activity was shown to increase in chloroquine resistant parasites under drug pressure [17]. Ferriprotoporphyrin IX, which is produced in large quantities during the digestion of hemoglobin by the parasite, has been characterized as uncompetitive inhibitor of PfGST [14]. A pharmacologic inhibition of PfGST is likely to impair the peroxidase, conjugation and heme binding activity of the enzyme. This - together with the unique structural features of the enzyme - makes PfGST, a most promising antimalarial drug target.

Conventionally the canonical GSTs are homo- or heterodimeric proteins with each subunit consisting of a thioredoxin like domain I fused to an all $\alpha$-helical domain II [8]. The active site is located at the cleft between the two domains, consisting of two binding sites: the G-site which binds reduced glutathione and the more variable $\mathrm{H}$-site, which can accommodate a variety of substrates [18]. The H-site of PfGST differs significantly from that of its human counterpart in being more accessible to solvent molecules and amphiphilic inhibitors [18]. Aim of the present study was to investigate the role of GSH in tetramer-dimer transition of PfGST, as well as in modulation of functional properties of the enzyme together with structural, activity and stability characterstics of the enzyme. Using size exclusion chromatography and protein cross-linking as well as fluorescence and CD spectroscopy in combination with enzyme activity assays we studied this unique feature of PfGST in detail. The structural and functional characterization of PfGST does contribute to our understanding of the parasites' biology; the evolution of GSTs and to targeted drug development against malaria.

\section{Results and discussion PfGST is stabilized as a tetramer}

We carried out detailed studies on the structural and functional properties of PfGST. Figure 1A shows the overexpression and purification of PfGST, which had the expected molecular mass of about $26 \mathrm{kDa}$ and a purity of about $95 \%$. The quaternary structure of PfGST was analyzed by SEC and glutaraldehyde cross-linking. On the Superde ${ }^{\mathrm{TM}} 200$ column the protein showed a single peak with a retention volume of $13.7 \mathrm{~mL}$ which corresponds to a molecular mass of $104 \mathrm{kDa}$, as calculated from the values obtained for the protein standards (Figure 1B). This suggests that the PfGST is indeed present as a tetramer. This observation was further confirmed by the glutaraldehyde cross-linking of PfGST, which showed the presence of a cross-linked protein band of a molecular mass of about $104 \mathrm{kDa}$ corresponding to the tetramer of the enzyme (Figure 1B, inset). These results confirm previous experiments, which showed that recombinant $P f G S T$ forms under native conditions non-covalently linked higher aggregates corresponding to $98 \%$ to tetramers [19]. To our knowledge PfGST is the only enzyme of the GST family, which is present as a tetramer rather than a dimer.

\section{Glutathione binding to PfGST modulates the conversion of inactive tetramer to active dimer}

GSTs catalyze the general reaction GSH + R-X $\rightarrow$ GSR + H$\mathrm{X}$. GST brings the hydrophobic or amphiphilic substrate into close proximity with GSH and activates the sulfhydryl group of GSH thereby allowing the nucleophilic attack on 

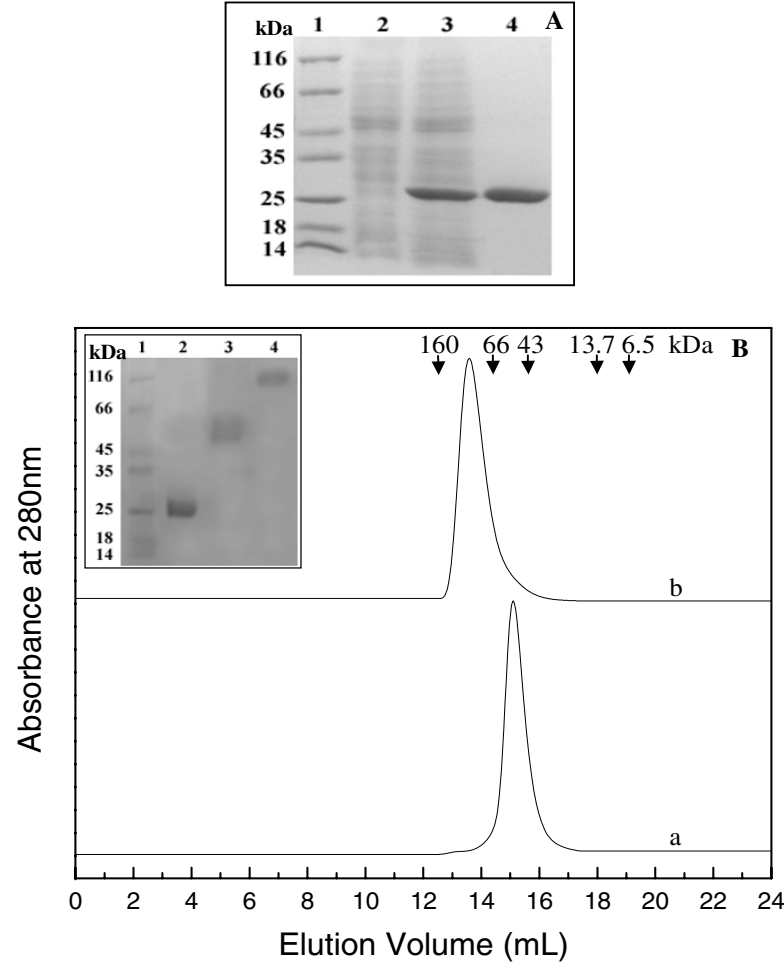

\section{Figure I}

Overexpression of PfGST in E. coli and purification of the recombinant protein over $\mathrm{Ni}-\mathrm{NTA}$ agarose. $\mathrm{A}$. SDS-PAGE analysis of cell lysate showing overexpression of PFGST and the purified protein. Lanes I-4 represent molecular weight markers, supernatant of un-induced culture lysate, supernatant of induced culture lysate and purified protein, respectively. B. Molecular weight andsubunit structure of PFGST dimer and tetramer. SEC profileof (a) dimeric PFGST; obtained by incubation of protein with $2 \mathrm{mM} \mathrm{GSH}$ and run with buffer containing $2 \mathrm{mM} \mathrm{GSH}$ and (b) tetrameric PfGST; incubated and run with buffer not containing GSH on a Superdex ${ }^{\mathrm{TM}} 20010 / 300 \mathrm{GL}$ column at $\mathrm{pH} 8.0$ and $25^{\circ} \mathrm{C}$. The column was calibrated with standard molecular weight markers: Glucose oxidase ( $160 \mathrm{kDa})$, albumin $(66 \mathrm{kDa})$, ovalbumin (43 kDa), ribonuclease $A(I 3.7 \mathrm{kDa})$ and aprotinin $(6.5 \mathrm{kDa})$. The curves have been displaced on $\mathrm{Y}$-axis for presentation. Inset shows the SDS-PAGE profile of glutaraldehyde crosslinked PfGST protein samples. Lanes I-4 represent molecular weight markers, uncross-linked native PfGST, glutaraldehyde cross-linked dimeric PfGST and tetrameric PfGST protein samples, respectively.

the electrophilic substrate [20]. All GSTs are highly specific towards GSH as the thiol substrate. GSH is bound in an extended conformation to the enzyme at the G-site via a network of specific polar interactions between the tripeptide and a number of protein moieties in domain I of one subunit and one or two amino acid residues in domain II of the other subunit of the dimer. Hence the dimer of the enzyme is the active conformation. As all GSTs for which this catalytic mechanism has been described are dimers we wanted to study if GSH binds to the tetrameric protein and if this tetramer is functionally active.

The binding of GSH to PfGST can be investigated by monitoring the intrinsic tryptophan fluorescence of the enzyme. The substrate binding results in partial quenching of the fluorescence due to direct interactions between the bound substrate and the indole fluorophore of the tryptophan moiety $[19,21]$. We monitored the tryptophan fluorescence of the PfGST tetramer incubated with increasing concentrations of GSH. Quenching of the tryptophan fluorescence intensity was observed at GSH concentrations between 0 to $0.8 \mathrm{mM}$ (Figure 2A). Further increase in GSH concentration did not lead to any further quenching of fluorescence. However, a maximum of only about $20 \%$ quenching of the tryptophan signal was observed under these conditions. No alteration in the emission wavelength maxima of the tryptophan fluorescence was observed in the presence of GSH. These observations collectively suggest that in presence of GSH the PfGST tetramer undergoes structural change.

A very interesting result was obtained when we studied the effect of GSH binding on the oligomeric structure of the enzyme. Figure 2B shows the SEC profiles of the PfGST tetramer after incubation with increasing concentration of GSH. With increasing concentrations of GSH, two populations of the enzyme, one corresponding to the tetramer (retention volume $13.7 \mathrm{~mL}$ ) and the other one corresponding to the dimer (retention volume $15.0 \mathrm{~mL}$; molecular mass about $52 \mathrm{kDa}$ ) were observed. Furthermore, with increasing concentrations of GSH an enhancement in the area under the peak corresponding to the dimer along with a concomitant decrease in that corresponding to the tetramer were observed. At GSH concentrations $\geq$ $0.7 \mathrm{mM}$ only a single peak corresponding to the dimeric protein was observed. This was further confirmed by the appearance of a cross-linked protein band of about 52 $\mathrm{kDa}$ (Figure 1B, inset). Furthermore, this GSH dependent tetramer to dimer transition was found to be independent of the enzyme concentration (data not shown). These observations demonstrate that GSH binding to the PfGST tetramer induces a dissociation of the tetramer into dimers and that in the presence of higher GSH concentrations a GSH-stabilized dimer is obtained.

As GSH was shown to induce dissociation of the native PfGST tetramer into dimers we carried out activity assays in presence of increasing concentrations of GSH to see which configuration, dimer or tetramer, was functionally active. Figure 2A shows the functional activity of the PfGST tetramer in the presence of increasing concentra- 

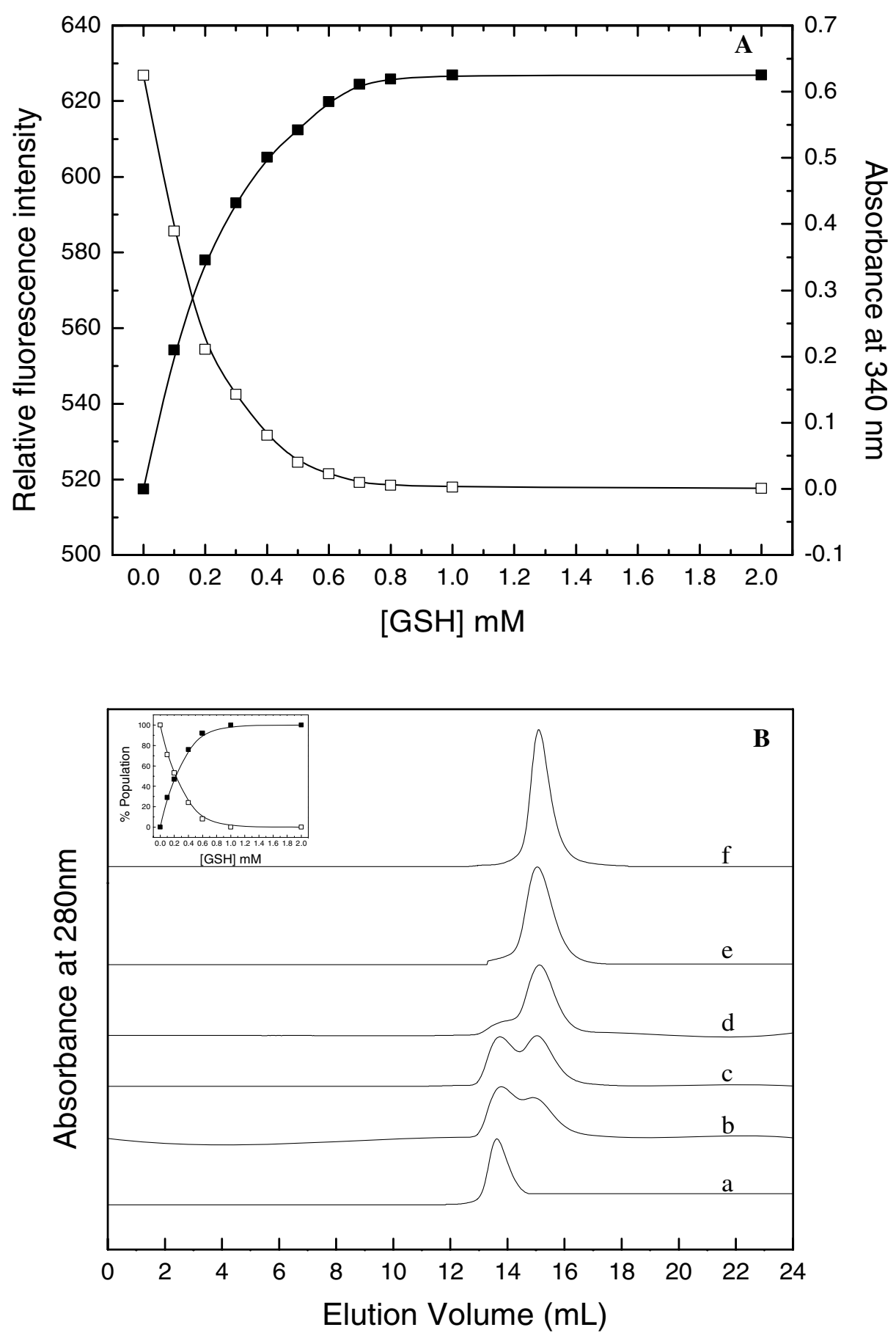

\section{Figure 2}

Effect of GSH on oligomeric status and enzymatic activity of PfGST. A. Quenching of tryptophan fluorescenceintensity and enzymatic activity of tetrameric PfGST withincreasing concentrations of GSH. In the figure, solid and hollow squares denote data for enzymatic activity and fluorescence, respectively. B. SEC profile of tetrameric PfGST incubated with increasing concentrations of GSH for $2 \mathrm{~h}$ at $25^{\circ} \mathrm{C}$ and run in the same GSH containing buffer. In the figure, a-f represent curves for tetrameric PfGST incubated with $0,0.1,0.2,0.4,0.6$ and $2.0 \mathrm{mM} \mathrm{GSH}$, respectively. The curves have been displaced on $\mathrm{Y}$-axis for presentation. Inset shows the percent population of dimeric (solid squares) and tetrameric (hollow squares) species of PfGST with increasing concentration of GSH based on the data obtained from the curves displayed in the main figure. 
tions of GSH. An exponential increase in enzymatic activity of PfGST was observed at GSH concentrations between 0 and $0.7 \mathrm{mM}$. However, further increase in GSH concentration did not lead to further enhancement in activity. The results demonstrate that the conditions for attainment of maximal activity, namely saturation of GSH binding to the PfGST tetramer as well as complete dissociation into dimers all occur at the same GSH concentration. This demonstrates that the dimer and not the tetramer of PfGST is the functionally active form of the enzyme. The fact that the increase in activity is directly paralleled by the dissociation of the tetramer indicates that the $\mathrm{K}_{\mathrm{m}}$ values determined do not simply reflect the affinity of GSH to the active enzyme but at the same time induce major changes in quaternary structure resulting in active enzyme. This is the first report for any GST where an alteration in oligomeric structure and not just small conformational change are observed upon GSH binding.

In direct comparison with $P f G S T$ we studied the effect of GSH on the oligomeric status of human GST. Figure 3 shows the SEC profiles of human GST in presence and absence of GSH. For human GST a single peak with a retention volume of $15.1 \mathrm{~mL}$ corresponding to a molecular mass of about $52 \mathrm{kDa}$ was observed which clearly represents the dimeric protein. For the enzyme sample incubated with GSH and run in presence of GSH no shift in retention volume from the column was observed. This was further confirmed by cross-linking of the eluted peaks (Figure 3 inset). This demonstrates that human GST is present as a dimer under physiological conditions and binding of GSH to the human GST dimer does not affect the oligomeric status of the enzyme.

From the recently reported crystal structure of PfGST, it has been deduced that in absence of ligands two biological dimers form a tetramer [18]. In the tetramer the homodimers are interlocked with each other by a loop interacting with the substrate-binding site (residues 113120). Upon binding of S-hexylglutathione, this loop undergoes rearrangement. The changed course of residues 113-120 in the liganded enzyme prevents locking of the dimers (19). A similar process is likely to take place upon GSH binding to the enzyme. The oligomerization of dimer into tetramer can be only a consequence and not the cause of the loss of affinity for GSH as the tetramer formation can be triggered by the structural change in the Gsite of the enzyme in the absence of GSH.

\section{The GSH-induced dissociation of the PfGST tetramer is a reversible process}

It has been reported that $P f G S T$ shows good stability and constant specific activity only when it is stored at $\geq 1 \mathrm{mM}$ GSH [21]. The specific activity of the enzyme stored in 10 $\mathrm{mM}$ GSH drops to $50 \%$ in a few minutes after SEC in

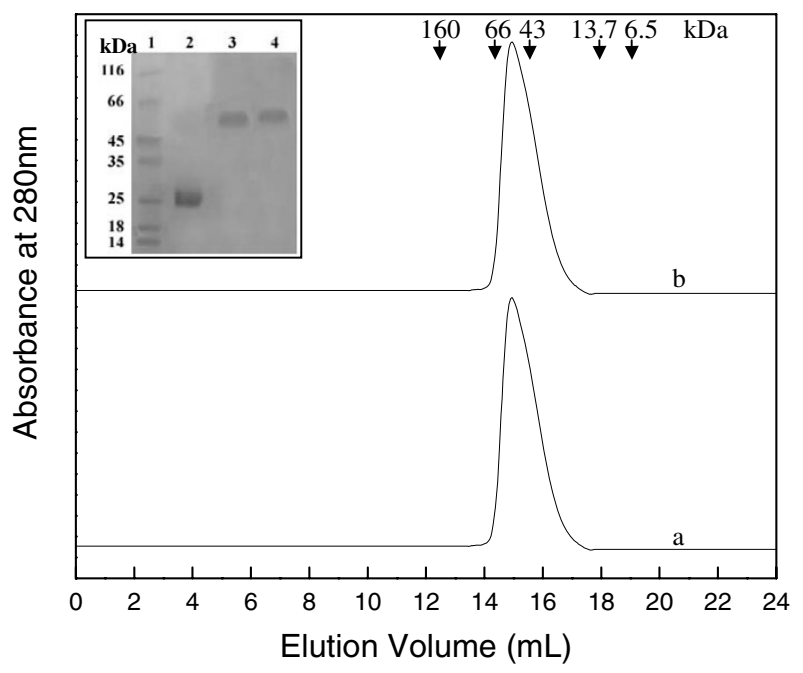

Figure 3

Effect of GSH on oligomeric status of human GST. SEC profile of human GST. Curve "a" represent hGST incubated and run with buffer containing $2 \mathrm{mM} \mathrm{GSH}$, while curve "b" represent $h$ GST incubated and run with buffer not containing GSH. The curves have been displaced on $\mathrm{Y}$-axis for presentation. Inset shows the SDS-PAGE profile of glutaraldehyde cross-linked $h$ GST protein samples. Lanes $I-4$ represent molecular weight markers, uncross-linked native $h$ GST, glutaraldehyde cross-linked $h$ GST incubated with GSH and hGST incubated without GSH protein samples, respectively.

absence of GSH or when the enzyme is stored in buffer where GSH is absent [21]. However, no reason for this partial or complete inactivation of PfGST has been given so far. Our results indicate that GSH is essential for the conversion of enzymatically inactive tetramer into active dimer. It might thus be possible that the tetramer-dimer conversion of $P f G S T$ is a reversible process, which is modulated by GSH. To study this hypothesis two sets of experiments were carried out. Firstly the tetrameric PfGST incubated with $2 \mathrm{mM}$ GSH was loaded on a Superdex ${ }^{\mathrm{TM}}$ 200 column and was run with buffer, which did not contain GSH. Figure 4A summarizes the results of this study. A SEC profile containing two populations, dimer and tetramer, of enzyme was observed under these conditions (Figure $4 \mathrm{~A}(\mathrm{e})$ ). This suggests that a significant population (about $75 \%$ ) of the enzymatically active dimer is converted into inactive tetramer under these conditions and this is what was reflected in the activity profile where only about 30\% activity was observed for the sample obtained after SEC (Figure 4B). In the second experiment, the GSHstabilized enzymatically active dimer was dialyzed against the buffer in which GSH was absent. The dialyzed protein sample was loaded on the Sephadex S-200 column after 4 and $24 \mathrm{~h}$ of dialysis. Figure $4 \mathrm{~A}(\mathrm{c}, \mathrm{d})$ summarizes the 
results. For the $4 \mathrm{~h}$ dialyzed sample, the presence of both the dimer and the tetramer in a ratio of about 1:3 was observed. However, after $24 \mathrm{~h}$ of dialysis only the tetrameric enzyme species was observed. The analysis of the enzymatic activity of these two samples showed about $28 \%$ and $0 \%$ activity, respectively (Figure $4 \mathrm{~B}$ ). These studies clearly demonstrate that the GSH-induced tetramer to dimer conversion of PfGST is a reversible process with the equilibrium shifting to either side due to the presence or the absence of GSH.
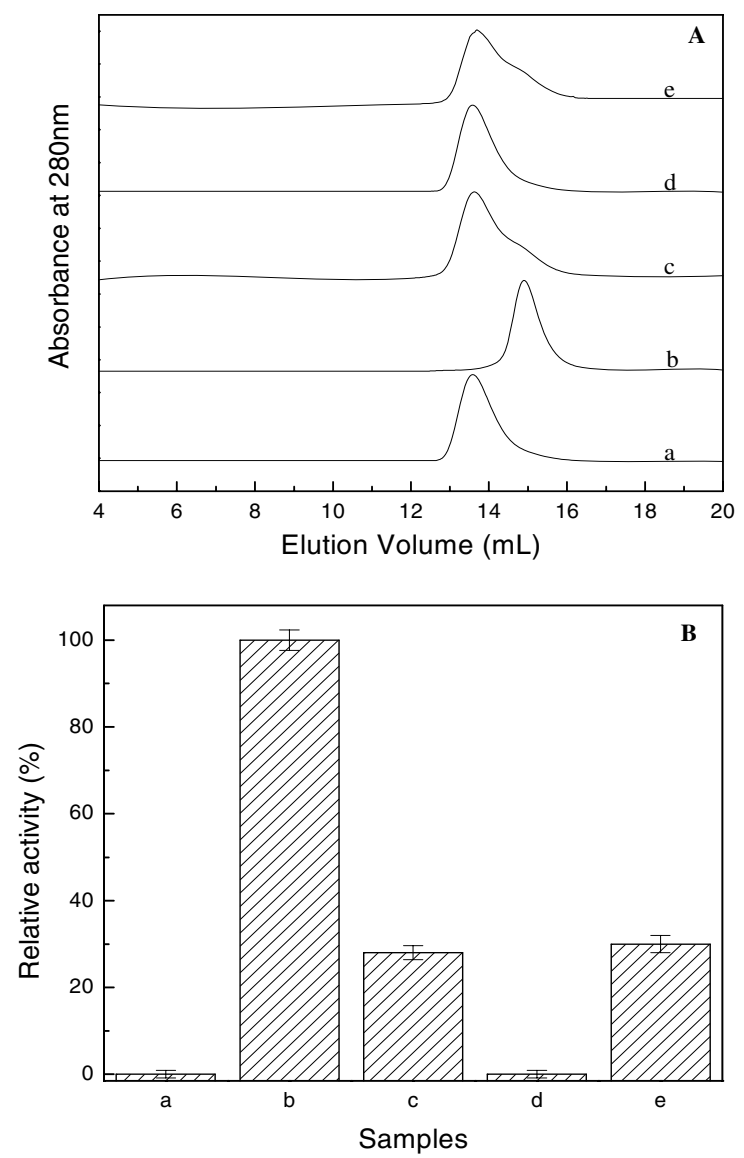

\section{Figure 4}

Deciphering the reversibility of the tetramer-dimer transition. A. SEC profile of PfGST. Curves a-e represent tetrameric PfGST, dimeric PfGST, dimeric PfGST dialysed for $4 \mathrm{~h}$ in buffer devoid of GSH, dimeric PfGST dialysed for $24 \mathrm{~h}$ in buffer devoid of GSH, dimeric PfGST loaded on the column equilibrated and run with buffer without GSH. The curves have been displaced on $\mathrm{Y}$-axis for presentation. $\mathrm{B}$. Enzymatic activity assay of the eluted peaks. In the figure, bars a-e represents data for the peaks described in $4 \mathrm{~A}$.

\section{Stability of PfGST tetramer and dimer against $\mathrm{pH}-$, GdnHCl- and urea-induced denaturation}

The effect of changing $\mathrm{pH}$ values on the structural features of the dimeric and tetrameric species of PfGST was studied. Figure 5A shows the changes in secondary structure of dimeric and tetrameric PfGST with varying $\mathrm{pH}$. For the PfGST dimer, the secondary structure was found to be stable between pH 5.0 and 8.0 as no alteration in secondary structure of the protein was observed. However, decrease in $\mathrm{pH}$ below 5.0 or increase in $\mathrm{pH}$ above 8.0 resulted in denaturation of the enzyme as indicated by a significant loss in secondary structure. At $\mathrm{pH} 4.0$ or below and $\mathrm{pH}$ 10.0, almost complete loss of secondary structure was observed. For the PfGST tetramer, a sigmoidal dependence of the loss of secondary structure on the $\mathrm{pH}$ value was observed. Between pH 10.0 and 6.0 no alteration was determined. However, decrease in $\mathrm{pH}$ below 6.0 resulted in loss of secondary structure. Interestingly, for the PfGST tetramer even at a $\mathrm{pH}$ as low as 3.0 , only a partial loss of secondary structure of about $50 \%$ was observed suggesting only partial unfolding of the enzyme at low $\mathrm{pH}$.

The $\mathrm{pH}$ dependent studies demonstrate that unlike the dimer which is denatured at alkaline $\mathrm{pH}$, the PfGST tetramer is resistant to alkaline $\mathrm{pH}$. Both the tetramer and the dimer show susceptibility to acidic $\mathrm{pH}$, the tetramer is however more stable under these conditions. Taken together these observations demonstrate that the PfGST tetramer is more stable than the dimer towards $\mathrm{pH}$ induced denaturation, which is likely to be due to stronger ionic interactions present in the tetrameric conformation.

The comparative unfolding and stability characteristics of PfGST tetramer and dimer were studied by monitoring the GdnHCl-induced changes in the structural properties of the proteins. To study the changes in the secondary and tertiary structure induced by $\mathrm{GdnHCl}$, far-UV CD and tryptophan fluorescence studies were carried out. A minimum time of about $2 \mathrm{~h}$ was found to be sufficient for achieving equilibrium under any of the denaturing conditions studied. Figure 5B summarizes the effect of increasing concentrations of $\mathrm{GdnHCl}$ on the $\mathrm{CD}$ ellipticity at 222 $\mathrm{nm}$ and tryptophan emission maxima of the PfGST tetramer and dimer. For both conformations of the enzyme, a sigmoidal loss of the CD signal at $222 \mathrm{~nm}$ and a shift in the emission wavelength maxima of the tryptophan fluorescence from $328 \mathrm{~nm}$ to $353 \mathrm{~nm}$ was observed. Furthermore, the changes in tryptophan fluorescence and the secondary structure of the proteins were found to be concomitant with respect to $\mathrm{GdnHCl}$ concentration. However, the $\mathrm{GdnHCl}$ concentration over which the transition was observed was different for the two forms of the enzyme. For the PfGST dimer, the transition occurred between 1.0 and 4.0 $\mathrm{M} \mathrm{GdnHCl}$, with $\mathrm{C}_{1 / 2}$ for the transition being $2.5 \mathrm{M} \mathrm{GdnHCl}$. For the PfGST tetramer 

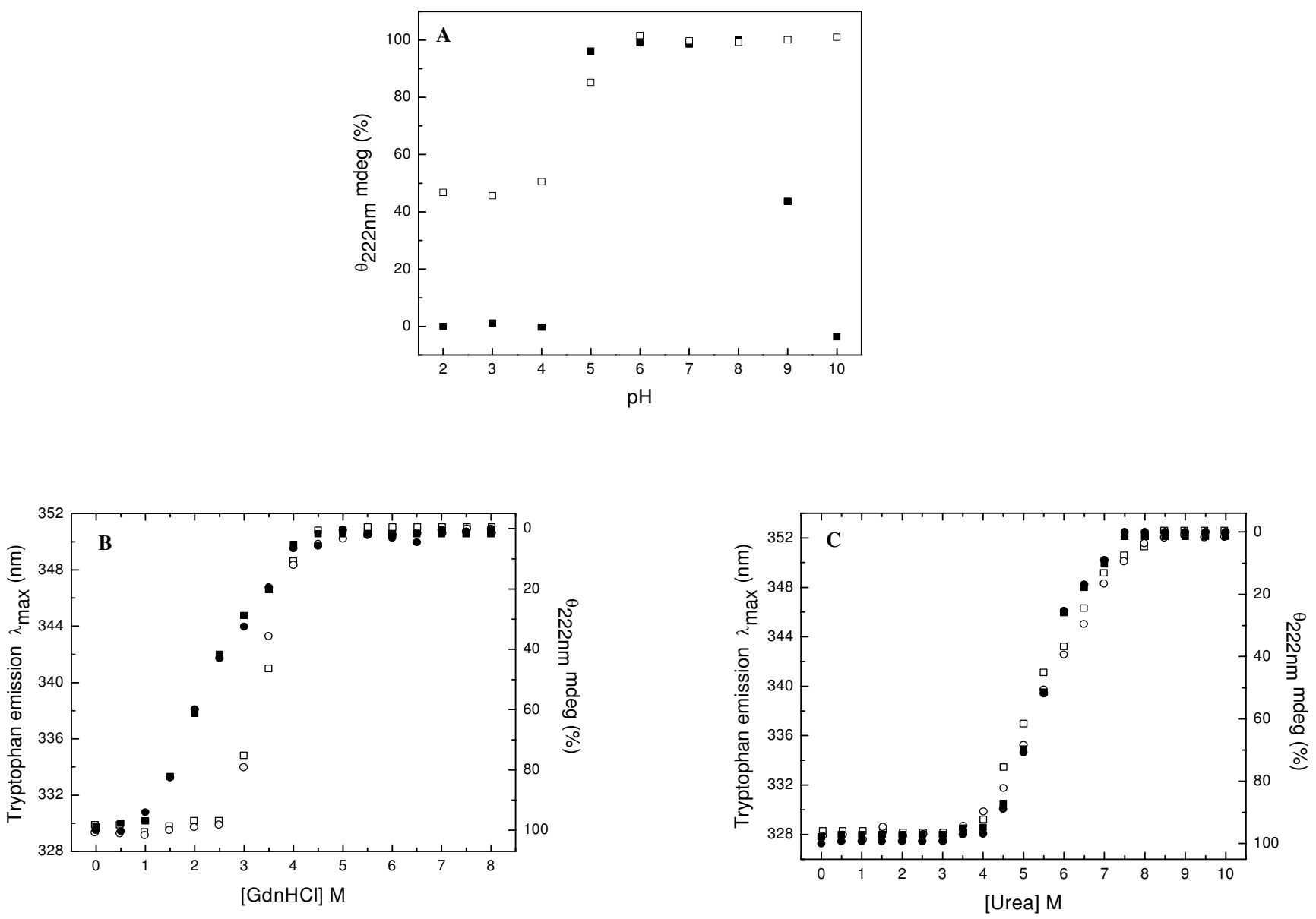

\section{Figure 5}

pH-, GdnHCl- and urea- induced unfolding of dimeric and tetrameric PfGST at pH 8.0 and $25^{\circ} \mathrm{C}$. A. Effects of the $\mathrm{pH}$ on the CD signal at $222 \mathrm{~nm}$ for the dimeric (solidsquares) and tetrameric (hollow squares) PfGST. The data is presented as percentage with the value observed for enzyme at $\mathrm{pH} 8.0$ taken as $100 \%$. B Effect of increasing $\mathrm{GdnHCl}$ concentrations on the CD ellipticity at $222 \mathrm{~nm}$ and the tryptophan emission wavelength maxima of dimeric and tetrameric PfGST. C. Effect of increasing urea concentrations on the CD ellipticity at $222 \mathrm{~nm}$ and the tryptophan emission wavelength maxima of dimeric and tetrameric PfGST. In the panel B and C, solid squares, solid circles, hollow squares and hollow circles represent data for CD of the dimers, fluorescence of the dimers, $C D$ of the tetramers and fluorescence of the tetramers, respectively. The CD data has been presented as percentage with the value observed in the absence of denaturant $(\mathrm{GdnHCl}$ or urea) taken as $100 \%$. The experimental details are mentioned in the Methods section.

the transition was observed between 2.5 and $4.0 \mathrm{M} \mathrm{Gdn-}$ $\mathrm{HCl}$, with $\mathrm{C}_{1 / 2}$ for the transition being $3.5 \mathrm{M} \mathrm{GdnHCl}$.

The comparative unfolding and stability characteristics of the PfGST tetramer and dimer with urea were studied by monitoring the urea-induced changes in the structural properties of the proteins. Figure 5C summarizes the effect of increasing concentrations of urea on the CD ellipticity at $222 \mathrm{~nm}$ and the tryptophan emission maxima of PfGST tetramers and dimers. For both conformations, a similar sigmoidal loss of the CD signal at $222 \mathrm{~nm}$ and a shift in the emission wavelength maxima of the tryptophan fluo- rescence from $328 \mathrm{~nm}$ to $353 \mathrm{~nm}$ was observed for CD and fluorescence spectroscopy, respectively. According to this data, $\mathrm{a} \mathrm{C}_{1 / 2}$ of about $5.5 \mathrm{M}$ urea was found to be associated with the unfolding of both the PfGST tetramer and dimer.

These results demonstrate that the PfGST tetramer has significantly higher stability against $\mathrm{GdnHCl}$ denaturation as compared to the PfGST dimer. However, with respect to urea denaturation the tetramer and the dimer showed similar stability. These observations along with the $\mathrm{pH}$ stability studies suggest that the higher stability of the 
tetramer of $P f G S T$ as compared to the dimer is probably due to the presence of stronger ionic interactions in this conformation of enzyme.

\section{Conclusion}

The glutathione S-transferase of the malarial parasite Plasmodium falciparum is the first GST for which a GSH induced tetramer-dimer transition has been described. Interestingly, the surrounding GSH levels in a physiological concentration range of $0-0.7 \mathrm{mM}$ regulate this transition. Until now the determination of GSH levels in malarial parasites has been restricted to late trophozoites, which represent the largest blood stage form and are thus accessible to studies. Meierjohann et al., described a variation in GSH levels between different strains [22]. For PfDd2 they measured $134 \mathrm{nmol} / 10^{10}$ cells, for Pf3D7 67 $\mathrm{nmol} / 10^{10}$ cells. Assuming that a parasitized erythrocyte has $90 \mathrm{fL}\left(90 \times 10^{-15} \mathrm{~L}\right)$ and $30 \mathrm{fL}$ (corresponding to $1 / 3$ of the host cell compartment as described in Atamna and Ginsburg [23]) of the cell is represented by the trophozoite parasite, $10^{10}$ parasite cells would have a volume of $30 \times 10^{-5} \mathrm{~L}$ which is equal to $300 \mu \mathrm{L} .134 \mathrm{nmol} / 300 \mu \mathrm{L}$ corresponds to $447 \mu \mathrm{M}$ for PfDd2. For Pf3D7 a value of $223 \mu \mathrm{M}$ can be calculated. In FCBR-strain, Luersen et al., found a GSH concentration of $0.42 \mathrm{mM}$ [24]. Atamna and Ginsburg measured a GSH concentration of $790 \mathrm{nmol} /$ $10^{10}$ cells in the strain FCR3, corresponding to $2.6 \mathrm{mM}$. This is by a factor of 10 higher than measured in the 3D7 strain. All these data indicate that the GSH concentrations may vary considerably between different strains. Furthermore, the reliable determination of intraparasitic GSH concentrations remains challenging - particularly due to the limited material and the complex isolation and washing procedures required. These are also the reasons why GSH levels throughout the complex life cycle of Plasmodium in man and mosquitoes have not yet been studied although they might vary considerably $[25,26]$. Taking into account GSH concentrations between $223 \mu \mathrm{M}$ and $2.6 \mathrm{mM}$ (see above) as well as the fact that GSH has not yet been determined in most of the parasite stages, a physiological role of PfGST activity regulation by GSH in the micromolar range should be seriously considered.

It is thus possible that $P f G S T$ is produced and - depending on the surrounding GSH concentration - present as inactive but more stable tetramer. With increasing GSH concentrations the enzyme becomes active, with decreasing GSH concentrations it tetramerises again (Figure 6) and this reversible transition regulates the functional activity of PfGST. Under conditions of oxidative stress in the parasite, GSH levels can be depleted [25] and this would prevent the dissociation of PfGST leading to accumulation of inactive tetramer. Other regulatory principles including low molecular weight ligands, post-translational modifications or protein-protein interactions might further con- tribute to the modulation of this phenomenon. Since PfGST currently represents one of the most attractive antimalarial drug targets and the reversible changes in the oligomerization state of the protein is likely to reflect a novel principle of GST regulation, we will address this phenomenon in further studies.

\section{Methods \\ Materials}

All chemicals used in the study were purchased from Sigma-Aldrich Chemical Co., USA, and were of highest purity available. The $h$ GST from human placenta was also obtained from Sigma. The water used for all studies was of triple distilled grade. Superdex ${ }^{\mathrm{TM}} 200$ 10/300 GL column was purchased from GE Healthcare Biosciences, USA, while Ni-NTA agarose was from Qiagen.

\section{Protein expression and purification}

Recombinant PfGST was overexpressed in E. coli M15 cells and purified as described earlier [14]. The ESI-MS and SDS-PAGE of the purified PfGST protein showed that the preparation was more than $95 \%$ pure. The protein was dialyzed against $100 \mathrm{mM}$ potassium phosphate buffer, $\mathrm{pH}$ 8.0 containing $1 \mathrm{mM}$ EDTA. Two different buffer systems were used-containing or not containing $2 \mathrm{mM} \mathrm{GSH}$.

\section{Size exclusion chromatography}

Gel filtration experiments were carried out on a Super$\operatorname{dex}^{\mathrm{TM}} 200$ 10/300 GL column (manufacturer's exclusion limit $600 \mathrm{kDa}$ for proteins) on an ÄKTA-FPLC (GE Health

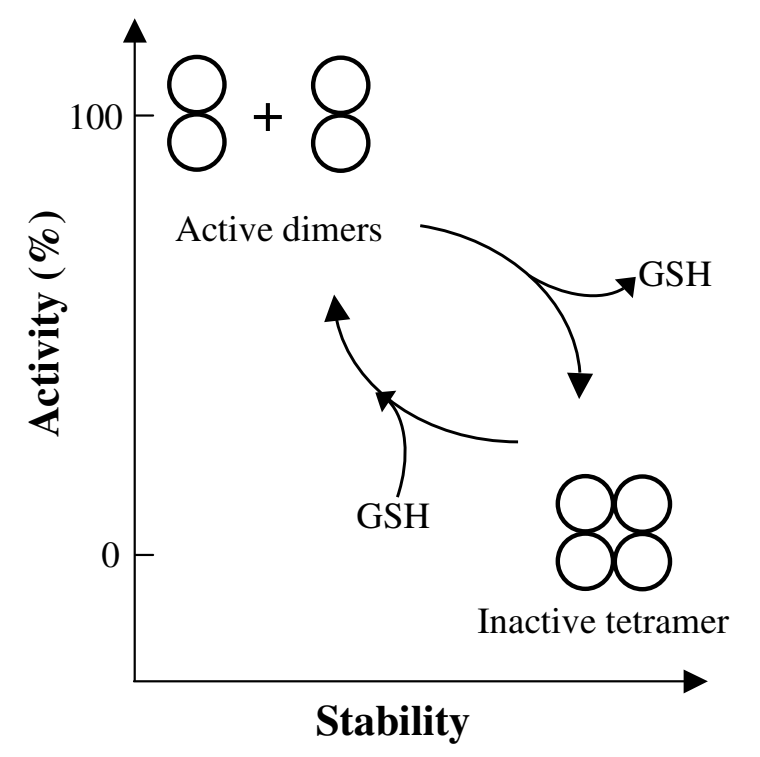

\section{Figure 6}

Schematic overview of PFGST tetramer-dimer transition with respect to GSH binding, activity and stability. 
Care Biosciences). The column was equilibrated and run with $100 \mathrm{mM}$ potassium phosphate buffer, $\mathrm{pH} 8.0$ containing $1 \mathrm{mM}$ EDTA, with a flow rate of $0.3 \mathrm{~mL} / \mathrm{min}$ at $25^{\circ} \mathrm{C}$. For GSH treated protein samples, the column was equilibrated and run with the above-mentioned buffer containing desired concentrations of GSH.

\section{Chemical cross-linking}

The protein samples, at a concentration of $50 \mu \mathrm{g} / \mathrm{mL}$, were used for cross-linking studies. An aliquot of 25\% (w/v) glutaraldehyde was added to obtain a final glutaraldehyde concentration of $1 \%$. The samples $(2 \mathrm{~mL})$ were stirred for $30 \mathrm{~min}$ at room temperature followed by quenching of the cross-linking reaction by the addition of $2 \mu \mathrm{L}$ of $\beta$ mercaptoethanol. After incubation for $20 \mathrm{~min}, 3 \mu \mathrm{L}$ of $10 \%$ aqueous sodium deoxycholate was added. The $\mathrm{pH}$ of the reaction mixtures was lowered to $2-2.5$ by the addition of orthophosphoric acid, which resulted in precipitation of the cross-linked proteins. After centrifugation, the obtained precipitates were re-dissolved and the molecular mass of the cross-linked products was determined by $10 \%$ SDS-PAGE.

\section{Enzymatic activity}

PfGST activity using GSH and CDNB as substrates was determined spectrophotometrically at $340 \mathrm{~nm}$ on the basis of the extinction coefficient for the product $S-(2,4$ dinitrophenyl) glutathione $\left(\varepsilon_{340 \mathrm{~nm}}=9.6 \mathrm{mM}^{-1} \mathrm{~cm}^{-1}\right)$. The assay mixture $(1 \mathrm{~mL})$ contained $2.0 \mu \mathrm{M}$ PfGST enzyme and $1 \mathrm{mM}$ GSH in $100 \mathrm{mM}$ potassium phosphate buffer, pH 8.0, 1 mM EDTA. The reaction was started by addition of $0.5 \mathrm{mM}$ CDNB. One unit of GST activity was defined as the conjugation of $1 \mu \mathrm{mol}$ of CDNB with GSH per minute at $25^{\circ} \mathrm{C}$ [14]. The data was recorded with a Perkin-Elmer Lambda $25 \mathrm{UV} / \mathrm{Vis}$ spectrophotometer at $25^{\circ} \mathrm{C}$.

\section{Fluorescence spectroscopy}

Fluorescence spectra were recorded with a Perkin-Elmer LS50B spectrophotometer in a $5 \mathrm{~mm}$ path length quartz cell. $2.0 \mu \mathrm{M}$ protein was used for the studies. The samples were excited at $285 \mathrm{~nm}$ and the emission spectra were recorded in the wavelength range of 300-400 $\mathrm{nm}$. Spectral scans were repeated thrice and the average was taken. The excitation and emission slits were kept as 8 and $6 \mathrm{~nm}$ respectively. Data was recorded at $25^{\circ} \mathrm{C}$.

\section{Far- ultraviolet circular dichroism}

Far- UV CD measurements were made with a Jasco J-810 spectropolarimeter calibrated with ammonium(+)-10camphor sulphonate. CD spectra were measured at an enzyme concentration of $2 \mu \mathrm{M}$ with a $1 \mathrm{~mm}$ cell at $25^{\circ} \mathrm{C}$. In a typical experiment 3 spectral scans were taken. The values obtained were normalized by subtracting the baseline recorded for the buffer having the same concentration of denaturant and/or GSH under identical conditions.

\section{pH denaturation}

Protein $(2 \mu \mathrm{M})$ was dissolved in $10 \mathrm{mM}$ citrate/glycine/ hepes buffer (containing $1 \mathrm{mM}$ EDTA) of varying $\mathrm{pH}$ (from 2.0 to 10.0) in the absence or presence of GSH and was incubated for $2 \mathrm{~h}$ at room temperature $(\mathrm{pH}$ of the solution maintained) before the measurements were made.

\section{$\mathrm{Gdn} \mathrm{HCl}$ and urea denaturation}

Protein $(2 \mu \mathrm{M})$ was dissolved in $100 \mathrm{mM}$ potassium phosphate buffer, pH 8.0 containing $1 \mathrm{mM}$ EDTA in the absence or presence of increasing concentration of Gdn$\mathrm{HCl} /$ urea with or without GSH. It was incubated for $2 \mathrm{~h}$ at room temperature, before the measurements were made.

\section{Abbreviations}

PfGST, Plasmodium falciparum glutathione S-transferase; hGST, human GST; GSH, Reduced glutathione; $\mathrm{C}_{1 / 2}$, Denaturant concentration at transition midpoint; $\mathrm{CDNB}$, 1-chloro-2,4-dinitrobenzene; ESI-MS, Electrospray ionization mass spectrometry; SEC, Size exclusion chromatography; Ni-NTA, Nickel-nitrilotriacetic acid; GdnHCl, Guanidine hydrochloride.

\section{Competing interests}

The author(s) declares that there are no competing interests.

\section{Authors' contributions}

TT carried out all the experiments and the data analysis. $\mathrm{SR}, \mathrm{KB}$ and $\mathrm{VB}$ conceived the study, participated in its design and coordination, and drafted the manuscript. All authors read and approved the final manuscript.

\section{Acknowledgements}

The study was supported by the Deutsche Forschungsgemeinschaft (BE 1540/4-4). TT wants to thank CSIR, New Delhi, for providing financial assistance.

\section{References}

I. Butler D, Maurice J, O'Brien C: Time to put malaria control on the global agenda. Nature 1997, 386:535-536.

2. Olliaro P: Mode of action and mechanisms of resistance for antimalarial drugs. Pharmacol Ther 2001, 89:207-219.

3. Hayes JD, Flanagan JU, Jowsey IR: Glutathione transferases. Annu Rev Pharmacol Toxicol 2005, 45:5I-88.

4. Mannervik B, Danielson UH: Glutathione transferases-structure and catalytic activity. CRC Crit Rev Biochem 1988, 23:283-337.

5. Bruns CM, Hubatsch I, Ridderstorm M, Mannervik B, Tainer JA: Human glutathione transferase A4-4 crystal structures and mutagenesis reveal the basis of high catalytic efficiency with toxic lipid peroxidation products. J Mol Biol 1999, 288:427-439.

6. Collinson EJ, Wheelar GL, Garrido EO, Avery AM, Avery SV, Grant CM: The yeast glutaredoxins are active as glutathione peroxidases. J Biol Chem 2002, 277:16712-16717.

7. Neuefeind T, Reinemer P, Bieseler B: Plant glutathione S-transferases and herbicide detoxification. Biol Chem 1997, 378: 199-205.

8. Salinas $A E$, Wong MG: Glutathione S-transferases-a review. Curr Med Chem 1999, 6:279-309.

9. Lyttle MH, Satyam A, Hocker MD, Bauer KE, Caldwell CG, Hui HC, Morgan AS, Mergia A, Kauvar LM: Glutathione-S-transferase 
activates novel alkylating agents. I Med Chem 1994, 37:150I-I507.

10. Meyer DJ, Coles B, Pemble SE, Gilmore KS, Fraser GM, Ketterer B: Theta, a new class of glutathione transferases purified from rat and man. Biochem J I99I, 274:409-4I4.

II. Pemble SE, Wardle AF, Taylor JB: Glutathione S-transferase class Kappa: characterization by the cloning of rat mitochondrial GST and identification of a human homologue. Biochem J 1996, 3 19:749-754.

12. Board PG, Baker RT, Chelvanayagam G, Jermiin LS: Zeta, a novel class of glutathione transferases in a range of species from plants to humans. Biochem J 1997, 328:929-935.

13. Frova C: Glutathione transferases in the genomics era: new insights and perspectives. Biomol Eng 2006, 23:149-169.

14. Harwaldt P, Rahlfs S, Becker K: Glutathione S-transferase of the malarial parasite Plasmodium falciparum: characterization of a potential drug target. Biol Chem 2002, 383:82I-830.

15. Liebau E, Bergmann B, Campbell AM, Teesdale-Spittle P, Brophy PM, Luersen K, Walter RD: The glutathione S-transferase from Plasmodium falciparum. Mol Biochem Parasitol 2002, I 24:85-90.

16. Deponte M, Becker K: Glutathione S-transferase from malarial parasites: structural and functional aspects. Methods Enzymol 2005, 40I:240-252.

17. Srivastava P, Puri SK, Kamboj KK, Pandey VC: Glutathione-Stransferase activity in malarial parasites. Trop Med Int Health 1999, 4:25I-254.

18. Fritz-Wolf K, Becker A, Rahlfs S, Harwaldt P, Schirmer RH, Kabsch W, Becker K: X-ray structure of glutathione S-transferase from the malarial parasite Plasmodium falciparum. Proc Natl Acad Sci 2003, 100:1382I-13826.

19. Hiller N, Fritz-Wolf K, Deponte M, Wende W, Zimmermann H, Becker K: Plasmodium falciparum glutathione S-transferasestructural and mechanistic studies on ligand binding and enzyme inhibition. Protein Sci 2006, I5:28I-289.

20. Pabst MJ, Habig WH, Jacoby WB: Glutathione S-transferase A. A novel kinetic mechanism in which the major reaction pathway depends on substrate concentration. J Biol Chem 1974 249:7|30-7|39.

21. Liebau E, De Maria F, Burmeister C, Perbandt M, Turella P, Antonin G, Federici G, Giansanti F, Stella L, Lo Bello M: Cooperativity and pseudo-cooperativity in the glutathione S-transferase from Plasmodium falciparum. J Biol Chem 2005, 280:26|2|-26I 28.

22. Meierjohann S, Walter RD, Muller S: Regulation of intracellular glutathione levels in erythrocytes infected with chloroquinesensitive and chloroquine-resistant Plasmodium falciparum. Biochem J 2002, 368:76 I-768.

23. Atamna $\mathrm{H}$, Ginsburg $\mathrm{H}$ : The malaria parasite supplies glutathione to its host cell - investigation of glutathione transport and metabolism in human erythrocytes infected with Plasmodium falciparum. Eur / Biochem 1997, 250:670-679.

24. Luersen K, Walter RD, Muller S: Plasmodium falciparuminfected red blood cells depend on a functional glutathione de novo synthesis attributable to an enhanced loss of glutathione. Biochem J 2000, 346:545-552.

25. Becker K, Rahlfs S, Nickel C, Schirmer RH: Glutathione-functions and metabolism in the malarial parasite Plasmodium falciparum. Biol Chem 2003, 384:55I-566.

26. Bozdech Z, Ginsburg H: Antioxidant defense in Plasmodium falciparum-data mining of the transcriptome. Malaria J 2004 3:23
Publish with Bio Med Central and every scientist can read your work free of charge

"BioMed Central will be the most significant development for disseminating the results of biomedical research in our lifetime. "

Sir Paul Nurse, Cancer Research UK

Your research papers will be:

- available free of charge to the entire biomedical community

- peer reviewed and published immediately upon acceptance

- cited in PubMed and archived on PubMed Central

- yours - you keep the copyright
BioMedcentral 